\title{
Forensic Analysis and the Inimitability of Human Footprints
}

\author{
Osisanwo F.Y. \\ Computer Science Department \\ Babcock University, Ilishan-Remo \\ Ogun State, Nigeria
}

\author{
Adetunmbi A.O, Alese B.K \\ Computer Science Department \\ Federal University Of Technology, Akure \\ Ondo State, Nigeria
}

\begin{abstract}
Divers features of human body are being explored on daily basis for person identification based on their uniqueness to individuals. The use of fingerprint in crime investigation has come to stay, but criminals are getting smarter by protecting their fingerprints from being evidence at a crime scene. Concealing of footprint or shoe print is yet to become popular, some criminals still go bare footed to crime scene. Footprint can be secured and analysed using image processing techniques such as de-noising, image partitioning, pattern matching This research looks at the inimitability of person footprints, should in any circumstance foot prints are found at a scene and are required to suggest a suspect or criminal. Image processing algorithms are employed to analyse barefoot prints in other to establish the uniqueness of the footprints.
\end{abstract}

\section{INTRODUCTION}

Forensic analysis is the use of controlled and documented analytical and investigative techniques to identify, collect, examine and preserve digital information. Meanwhile these analyses can also be the adopted in investigation of scenes of crime, where various evidences are left behind.

Forensic experts adopt diverse techniques to analyse evidence that are found at crime scene. The technique to adopt is determined by the type of evidence found; the evidence could be physical, trace, or biological. Some of the techniques include the image processing technique. This is used when evidence found can be registered as an image such as face evidence, fingerprint, tire tracks, foot print or shoe prints. Biological evidences that are from body features can be used to identify the person whose feature it is.

The process of using the footprint evidences for investigation or examinations consists of two processes: first the recovery process, which includes the discovery and preservation of the prints, and the second, the identification process, which involves evaluation, comparisons and findings related to the recovered impression Grieve 1988. As cited by [17]

The identification process involves the use forensic analysis techniques for analysis and evaluating the footprint for identification, this is done by first taking in the foot print as image for processing.

Image processing is a rapid growing aspect of computer science. Its growth has played a major role in the analysis of images or prints for forensics analysis. Image processing is a subset of the electronic domain or digital signal processing whereby an image is converted to an array of small integers, called pixels, representing a physical quantity such as scene radiance, stored in a digital memory, and processed by computer or other digital hardware [12]. Image processing can be defined as a process of extracting meaningful and useful information from an image [2].

An image is represented by a rectangular array of integers. An image $f(x, y)$ is divided into $N$ rows and $M$ columns. The intersection of a row and a column is termed a picture element (pixel). The number at each pixel represents the brightness or darkness (generally called intensity) of the image at that point. This implies that a pixel represents a value of either light intensity or colour [4][11].

Digital image processing is concerned primarily with extracting useful information from images; it starts from one image and produces a modified version of that image. This according to [10] involves the usage of complex algorithms to process the image before further extraction of useful information.

Foot prints to forensic experts when captured are taken as images and are treated as such for analysis and person identification, the prints are checked against database of footprint for any match in pattern.

\section{RELATED WORKS}

A lot of research work has been carried out on footprint recognition and also barefoot morphology [1][3][5][7][14] [15]. However, regardless of this the field of human identification based on footprint is still in embryonic stage. This is perhaps because existing work on footprint tracking and recognition is limited [15]. Also in some of the existing work, the approach used in footprint recognition involved manually sifting through a large database of captured foot prints, trying to find a match with the print found at the crime scene [3].

Subsequently, this research proposes a probable faster and easier means of electronically sifting through a 
database of barefoot print to find a match of any acquired footprint. The feature on the foot print that this work focuses on is the weight bearing areas of the foot print. As this is different with individuals.

\section{BAREFOOT MORPHOLOGY}

Barefoot Morphology is the structure of a foot. Research has shown that there are many factors which influence changes in the size and morphology of the foot such as: sex, weight, pregnancy, nutrition, age, genetics, disease, regional and ethnic variations due to heredity, and various environmental conditions such as climate [1]

Barefoot morphology comparison refers to the examination of the weight bearing areas of the foot [5]. The weight bearing area of a foot is determined by the part that bears largest concentrate of the body weight. This can be determined by first studying the structure of a foot.

According to [13], the human foot is a highly developed, biomechanically complex structure that serves to bear the weight of the body as well as forces many times the weight of the human body during momentum. There are about 26 bones in the human foot that provide structural support. Considering the variation in different individuals' bone heaviness or weight, body weight, bone pattern or shape resulting from deformity or diseases, individuals have unique patterns to the weight-bearing part of the foot. Research indicates that no two people have the same foot shape [9]. The foot can be subdivided into the hind foot, the mid foot, and the forefoot. The hind foot is composed of the ankle bone.

The mid foot contains five irregular bones that form the arches of the foot, which serves as a shock absorber. The forefoot is composed of five toes [6].

Standing on the feet, gives a foot prints that shows the weight bearing areas of the foot; either the forefoot, mid foot or hind foot. This is unlike the print taken in a seated position which bears little or no weight [8]. There are three types of arch height that are associated with human feet this determine how an individual works as well as the kind of print each foot makes. The arch heights include normal arch, high arch and flat arch. The arch height varies amongst individuals, hence the variation in shape and print of feet [8].

For the purpose of enhanced barefoot comparisons, this research is proposing foot comparison that is done bearing in mind the arch type of a foot, segmenting the foot print into the hind, the mid and fore foot, before extracting and comparing the weight bearing areas of different feet for match.

\section{IMAGE PROCESSING ALGORITHM}

These are the algorithms use for processing images. These are done in phases after the image had been acquired. The various phases for the processing includes noise removal, image partitioning or segmentation, feature extraction and finally pattern matching. This paper adopts all the phases.

\section{A. Noise Removal}

Footprints images are prone to different kinds of noise, which are introduced in divers' ways, either at acquisition point or point of transfer. They are threats to the real image, hence the importance of noise removal. This paper adopts the use of mathematical morphology operations for noise removal from the footprint. Mathematical Morphology operations are based on: Erosion and Dilation [16].

Morphological Opening is the operation used to de-noise the footprints, this is simply an erosion process followed by dilation.

The opening is expressed as follows

$X \circ B=(X \ominus B) \oplus B \quad \ldots 1$

Where $X$ is the image, $B$ is the structuring element also known as kernel, $\ominus$ is the dilation, $\oplus$ is the erosion.

\section{B. Image Partitioning}

After the noise removal, the image is partitioned into segments. With the aim of considering the intensity value of each sub region, the foot prints were partitioned into the regions of the foot; the hind, fore and mid foot. The following line of code was used for the partitioning: footSubimage=mat $2 \mathrm{cel} 1$ (newFootPrint, $[\mathrm{xF} 2, \mathrm{xF} 2, \mathrm{xF} 2],[\mathrm{yF} 2, \mathrm{yF} 2])$;

\section{C. $\quad$ Feature Extraction and Matching}

The feature considered is the intensity value of the print on the regions. The region bearing the greater weight is meant to have the higher intensity value. The pixel values assigned to each region are measured for the region with higher intensity. Then the Standard deviation of the pixel values of each region was calculated

$$
\begin{aligned}
& \sigma=\sqrt{\sum_{1=0}^{N-1}\left(\left(x_{i}-\mu\right)^{2}\right) /(N-1)} \\
& \mu \text { is the mean } \\
& x \text { is the image and } N \text { is the total number of values. }
\end{aligned}
$$

The values are then plotted in a graph against the each region label. For matching a test print is now compared with other foot print in the generated database.

\section{METHODOLOGY}

To acquire the foot prints for the analysis, volunteers were invited to step on an ink pad and then on a cardboard paper to make their footprint. Then the prints were scanned using a mercury scanner 2000c into the system for further analysis.

The use of Matrix Laboratory (Matlab) was employed by this research for the implementations of morphological opening for noise reduction, image partitioning, feature extraction and intensity matching.

\section{EXPERIMENTAL RESULTS}

\section{A. Experimental Results on De-noising}

The scanned prints are de-noised using the morphological opening. Figure 1 below show the foot print the original and the de-noised print. 


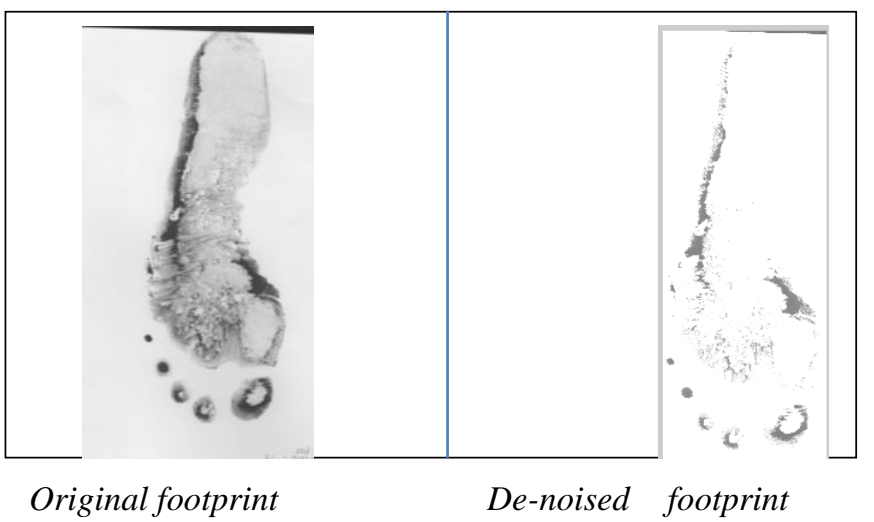

Figure 1 showing the original footprint and the de-noised

footprint using Morphological Opening

\section{B. $\quad$ Experimental Result on Partitioning}

After the de-noised image had been partitioned the resulting output is shown in figure 2 below

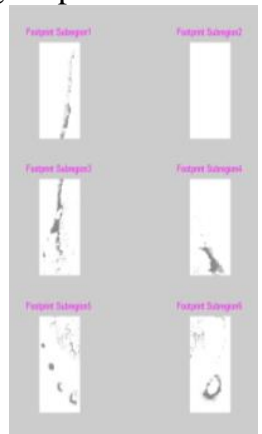

Figure 2 Partitioned foot print with each region labelled

\section{Experimental Result on Matching}

A test foot was now trained against other foot in the database. Below are just three categories of the output found for matching the test print with foot print in the database.

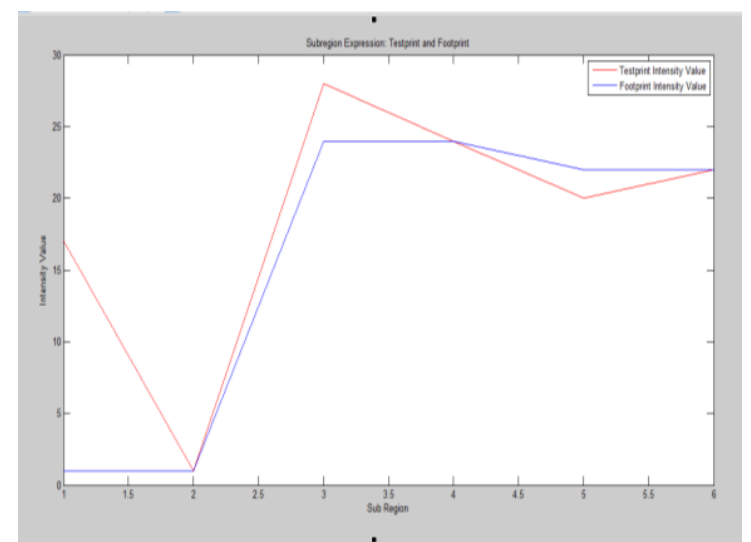

Figure 3: Intensity comparisons between prints using graph

Figure 3 above shows the graph representation of intensity comparison of the regions in the Test print with one

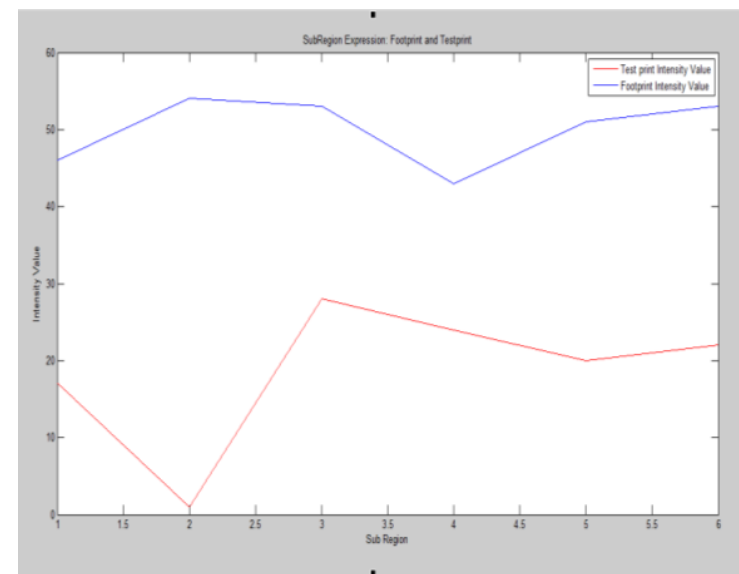

Figure 4: Intensity comparisons between prints using graph

Figure 4 shows the comparison between the test print and another foot print that does not have any similarity in their intensity values.

Meanwhile figure 5 below shows the comparison of the test print with itself giving an output that has same intensity at same point.

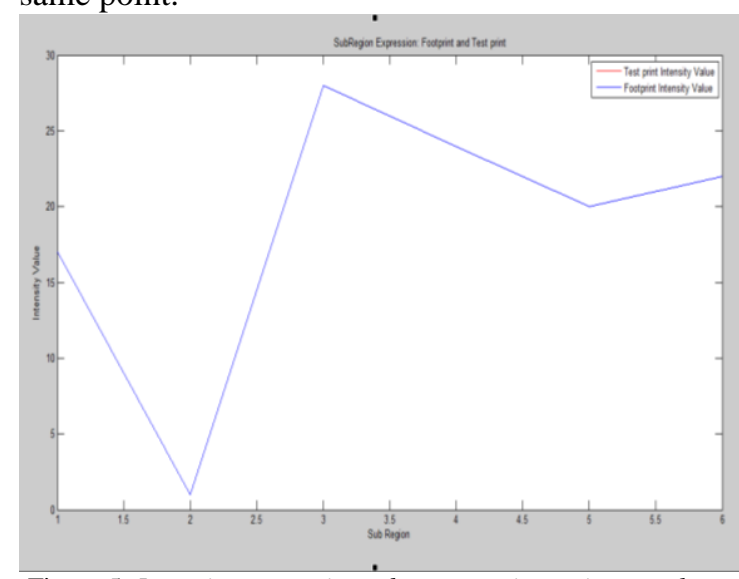

Figure 5: Intensity comparisons between prints using graph

of the print in the database. It can be deduce from the graph that the test print is not the same as the footprint. They only have similar values at region 2,3 and 6 .

\section{CONCLUSION}

This paper adopted mathematical morphology, image partitioning (resizing) and graph representation to analyse barefoot print for person identification. This proposed system cannot work as a standalone system for person identification but as a support system. It can be adopted for use by organisation or forensics experts, but the data capturing must be done carefully, so that additional pressure will not be introduced that will make the acquired data inconsistent for analysis as the intensity value is feature needed for comparison.

\section{ACKNOWLEDGEMENTS}

Our profound acknowledgements is directed first to all the volunteers who made the data collection possible by giving their footprints not minding the mess from the inkpad they had 
to step on. Secondly, to all researchers and various authors that made available information that aided this research work.

\section{REFERENCES}

[1] Brenda M. A. Rohren, M.A (2009).” Estimation of Stature from Foot and Shoe Length: Applications in Forensic Science" Department of Forensic Science, 5000 Saint Paul Avenue, Lincoln, Nebraska Nebraska Wesleyan University

[2] Elias O.G., Olajide M.S., Olaleke J.O. and Adetunmbi A.O. "Processing Biomedical Images Using Low Level Algorithm", Proceedings of the Second International Conference on Mobile e-Services, volume 2

[3] Hussey J. (2009) "Shoeprint Recognition, an Introduction" University of Edinburgh, School of Informatics, retrieved on June 02, 2012 from http://homepages.inf.ed. ac.uk/rbf/CVOnline/local_copies/AV0809/

[4] Ian T. Young,Jan J. Gerbrands and Lucas J. Van Vliet (1998). "Fundamentals of Image Processing." Delft University of Technology, Netherlands.

[5] Kennedy R.B. and Yamashita A. B. (2007) "Barefoot Morphology Comparisons: A Summary" Journal of Forensic Identification volume 57 issue 3 pages $383-413$

[6] Podiatry Channel (2014) "Anatomy of foot and Ankle"

[7] Robert B. Kennedy, Sanping Chen, Irwin S. Pressman, A. Brian Yamashita, and Ari E. Pressman (2005) "A LargeScale Statistical Analysis of Barefoot Impressions" Journal of Forensic Science, Sept. 2005, Vol. 50, No. 5

[8] Running Warehouse (2014) "Foot Analysis- Foot Motion Characteristics" retrieved on 25th August, 2014 from http://www.runningwarehouse.com/learningcenter/footanal ysis.html

[9] Ramsland K. (2014) “All about Fingerprints and other impression", retrieved August 25 , 2014 form Crime library http://www.crimelibrary.com/criminal_mind/forensics/fing erprints/9.html

[10]Seamann, T. (2003). Digital Image Processing Using Local Segmentation. Ph.D Thesis, School of Computer Science and Software Engineering, Faculty of Information Technology Monash University, Australia

[11]Shih Frank (2010) "Image Processing and Pattern Recognition", Institute of Electrical Electronic Engineers, Inc.

[12]Silver, B. (2000). An Introduction to Digital Image Processing. Cognex Corporation, Modular Vision System Division Natick

[13]Vinod K. P (2013) "Foot Anatomy", in Medscape- Drug and Disease edited by Thomas R. G. retrieved August 25th, 2014 from http://emedicine.medscape.com/ article/1922965-overview

[14 Yamashita A.B. (2007) “Forensic Barefoot Morphology Comparison" Canadian Journal of Criminology and Criminal Justice, volume 49, N0.5 pp 647-656

[15] Zhang J., Qian G., Kidane A (2009) "Footprint Tracking and Recognition Using A Pressure Sensing Floor", in conference proceedings of IEEE international conference on Image Processing, Cairo, Egypt

[16] Fisher R., Perkins A., Walker and Wolfart E.(2013) "Morphology" retrieved on 25th of August, 2014 from http://homepages.inf.ed.ac.uk/rbf/HIPR2

[17] DWAyne S. H. (2000) "FoOtwear, THE Missed EVIDENCE", ClPE LEAD LATENT PRINT EXAMINER SCOTTSDALE POLICE CRIME LAB. 